\title{
Ribosomes from Trypanosomatids: Unique Structural and Functional Properties
}

\author{
Maximiliano Juri Ayub, Walter J. Lapadula, \\ Johan Hoebeke and Cristian R. Smulski
}

Additional information is available at the end of the chapter

http://dx.doi.org/10.5772/48336

\section{Introduction}

Trypanosomatids are a monophyletic group of protozoa that diverged early from the eukaryotic lineage, constituting valuable model organisms for studying variability in different highly conserved processes including protein synthesis. Moreover, several species of trypanosomatids are causing agents of endemic diseases in the third world. There are many evidences suggesting that translation in these organisms shows important differences with that of model organisms such as yeast and mammals. These unique features, which have a great potential relevance for both basic and applied research, will be discussed in this chapter.

\section{Structural analysis}

\subsection{Cryo-electron microscopy map of Trypanosoma cruzi ribosome:} Unique features of the rRNA

Using the cryo-electron microscopy (cryo-EM) technique, a $12 \AA$ resolution density map of the T. cruzi $80 \mathrm{~S}$ ribosome has been constructed [1]. The overall structure of the T. cruzi $80 \mathrm{~S}$ ribosome exhibits well defined small (40S) and large (60S) subunits (Figure 1). Some of the landmark characteristics of the ribosome structure can be identified in the density map. Compared with the $80 \mathrm{~S}$ ribosome from yeast, both the small and large ribosomal subunits from T. cruzi are larger, mainly due to the size of the ribosomal RNA molecules. T. cruzi rRNA (18S rRNA: 2,315 nt and 28S rRNA: 4,151 nt) is one-fifth larger than yeast rRNA (18S rRNA: 1,798 nt; 25S rRNA: 3,392 nt) in total number of nucleotides.

Although the T. cruzi $80 \mathrm{~S}$ ribosome possesses conserved ribosomal structures, it exhibits many distinctive structural features in both the small and large subunits. Compared with 
other eukaryotic ribosomes, the T. cruzi ribosomal $40 \mathrm{~S}$ subunit appears expanded, due to the addition of a large piece of density adjacent to the platform region (Figure 1). As can be seen in the secondary structure of the T. cruzi 18S rRNA (Figure 2), this extra density must be attributed to two large expansion segments (ES) in domain II of the 18S rRNA, ES6 and ES7, designated as insertions of helices 21 and 26. These are the two largest ES in the T. cruzi 18S rRNA, involving 504 and 147 nucleotides, respectively.
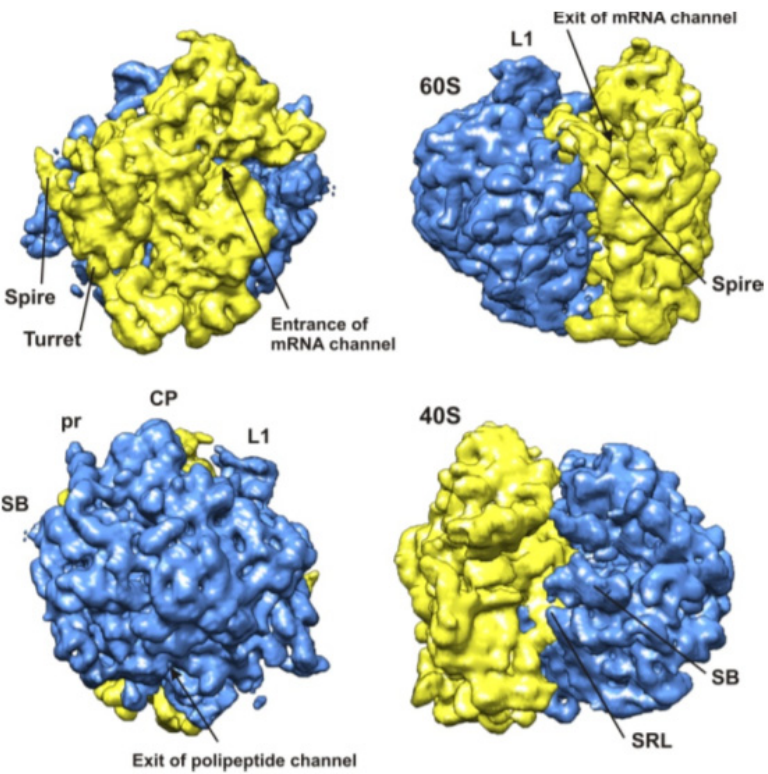

Figure 1. Cryo-electron microscopy map of T. cruzi $80 \mathrm{~S}$ ribosome. Blue: large subunit. Yellow: small subunit. Landmark characteristics are indicated: SB, stalk base; SRL, sarcin-ricin loop; L1, L1 protein; $\mathrm{CP}$, central protuberance; pr, prong.

Part of ES6/ES7 makes up a large helical structure (named the "turret"), located at the most lateral side of the $40 \mathrm{~S}$ subunit (Figures 1 and 2). The turret measures $205 \AA$ in length and forms the longest helical structure ever observed in a ribosome. The upper end of the turret appears as a sharp, freestanding spiral of $50 \AA$ in length, named "spire," located next to the exit of the mRNA channel. The distance between the spire and the mRNA exit is $\sim 130 \AA$. The lower portion of the turret extends all of the way to the bottom of the $40 \mathrm{~S}$ subunit. At its lower end, it bends by almost $90^{\circ}$ and forms a bridge with the $60 \mathrm{~S}$ subunit. This is a unique type of connection between the small and large subunits, as compared with all other ribosomal structures investigated to date [2]. Apart from the turret, the extra density in the 40S subunit also includes several small helical structures as part of ES6 and ES7. These helical structures observed in the density map are in accordance with the comparative analysis result based on ES6 sequences from $>3,000$ eukaryotes, in which several helices were identified only in kinetoplastida [3]. The ES3, ES9, and ES10 are located near helices 9, 39, and 41, respectively, and are associated with three small masses in the density map of the $40 \mathrm{~S}$ ribosomal subunit, one at the bottom of the $40 \mathrm{~S}$ ribosomal subunit, the other two in the head region. 


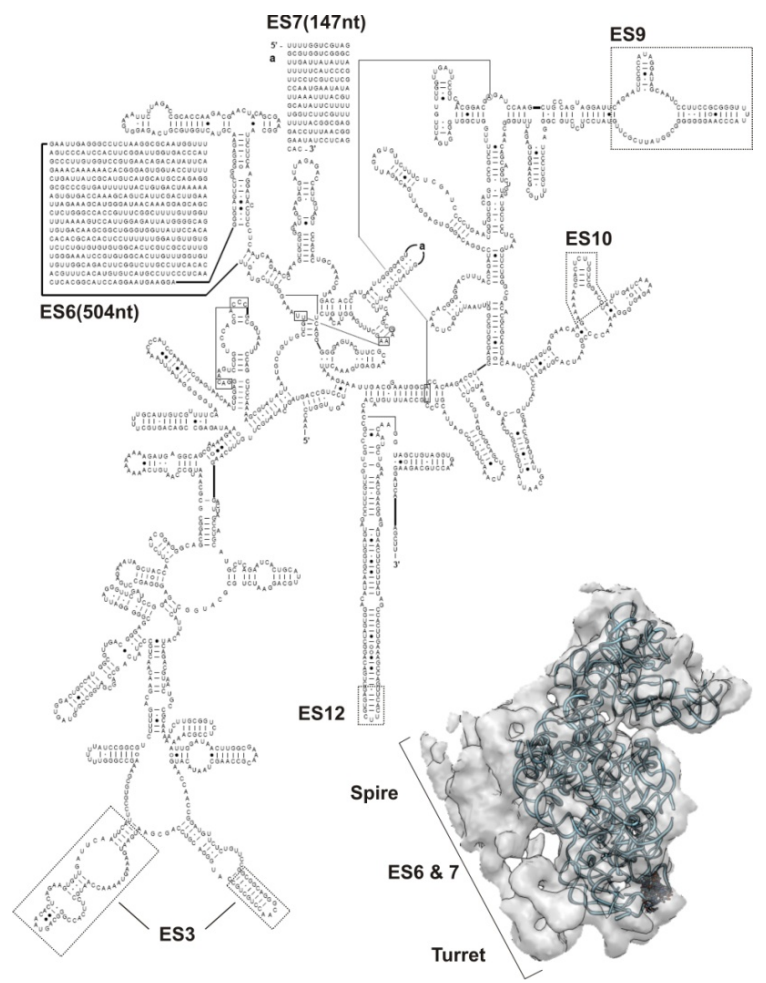

Figure 2. Secondary structure of T. cruzi $18 \mathrm{~S}$ rRNA with the characteristic ES. The $40 \mathrm{~S}$ subunit was superposed with the crystallized S. cerevisiae 18S rRNA (PDB: 3U5E). The volume occupied by ES6 and ES7 is indicated.

In contrast to the other rRNA regions, ES12, located in the long penultimate helix from $18 \mathrm{~S}$ rRNA, is shorter in T. cruzi compared to other eukaryotes. This results in the helix 44 in T. cruzi (113 nt) being longer than in E. coli (103 nt), but shorter than in yeast (129 nt). Consistently with these variations in length, the span of the density attributable to helix 44 in the T. cruzi ribosome also has an intermediate position between E. coli and yeast. Interestingly, this region forms the decoding center, and is also the action site of aminoglycoside antibiotics. Moreover, differences in this region have shown to be responsible for the higher susceptibility of trypanosomatid ribosomes to the aminoglycoside paromomycin [4], as will be discussed below.

The T. cruzi 60S subunit contains several large extra densities located at its periphery, in contrast with the large ribosomal subunit in yeast. The most common structures can be identified, such as the central protuberance (CP), L1 stalk, and stalk base of P proteins, as well as the conserved rRNA core structure (Figure 1). Although the secondary structure of the T. cruzi rRNAs in the 60S subunit is not available, the locations of most of the observed extra densities are consistent with the general locations of the rRNA expansion segments, which are, as a rule, at the surface of eukaryotic ribosomes. Among the extra densities, there is a large helical structure ("prong") located between the CP and helix 38, in the back of the 
$60 \mathrm{~S}$ subunit (Figure 1, pr). Interestingly, a similar feature was reported only in the structure of the human ribosome, but not in yeast neither bacterial ribosomes [5]. Morphological comparison of the $60 \mathrm{~S}$ ribosomal subunit from $T$. cruzi with those from yeast and higher eukaryotes reveals that the T. cruzi 605 ribosomal subunit does not possess the universal eukaryotic feature of a planar surface near the exit site of polypeptide [2]. Instead, the 605 ribosomal subunit from $T$. cruzi presents a shape that is similar to those from bacteria. In contrast to the conserved eukaryotic rRNA core structure, the location of the L1 stalk in $T$. cruzi, which is on one side of the $\mathrm{CP}$, does not match either of the two reported positions in yeast, known as the "in position" and "out position," in relation to the ratchet-like subunit rearrangement. Instead, the L1 stalk in T. cruzi takes an in-between position, possibly due to its high mobility. On the other side of the $\mathrm{CP}$, the $\mathrm{P}$ protein stalk is not visible in the density map of the T. cruzi 60S ribosomal subunit, whereas Western blots of the ribosome preparation using monoclonal antibodies against $\mathrm{P}$ proteins $(\mathrm{P} 0 / \mathrm{P} 1 / \mathrm{P} 2)$ showed that these proteins were present in the ribosome preparation. As homologs of the bacterial moiety L10/(L7/L12)4, P proteins are known to be very flexible, and the absence of a stalk in the cryo-EM density map is likely due to the lack of stabilization. A complete description of $T$. cruzi stalk region, components, interactions and complex formation will be discussed below.

\subsection{Sequence and proteomic analysis: Differences on the ribosomal proteins}

The Cryo EM map of T. cruzi ribosomes exposed important differences in comparison with the corresponding organelles of model organisms such as S. cerevisiae and mammals [1]. Some of them were attributed to large expansions in the primary sequence of the ribosomal RNA molecules. However, the presence of specific features due to ribosomal proteins is difficult to demonstrate by this technique. Therefore, using the $S$. cerevisiae ribosomal protein sequences as probes, it was possible to identify in the T. cruzi genome database all homologue genes [6]. The average amino acid identity between the S. cerevisiae and T. cruzi ribosomal proteins was remarkably low $(\sim 50 \%)$, taking into account the high degree of conservation of the ribosome through evolution.

The ribosomal proteins inferred by data mining were compared to the MS analysis results from whole parasites [7] and purified ribosomes [6]. Results are summarized in Tables 1 and 2 for proteins from the large and small subunits, respectively.

\begin{tabular}{|c|c|c|c|c|c|c|}
\multicolumn{2}{c|}{ S. cerevisiae } & \multicolumn{4}{c|}{ T. cruzi } \\
\hline Prot & 3U5E & Length (aa) & Length (aa) & $\%$ ID & Prot MS & Ribo MS \\
\hline L1 & - & 217 & 214 & 51 & + & - \\
\hline L2 & + & 254 & 260 & 62 & + & + \\
\hline L3 & + & 387 & 428 & 57 & + & + \\
\hline L4 & + & 362 & 374 & 49 & + & + \\
\hline L5 & + & 297 & 309 & 49 & + & + \\
\hline L6 & + & 176 & 193 & 43 & + & + \\
\hline L7 & + & 244 & 242 & 41 & + & + \\
\hline L8 & + & 256 & 315 & 47 & + & + \\
\hline
\end{tabular}




\begin{tabular}{|c|c|c|c|c|c|c|}
\hline \multicolumn{3}{|c|}{ S. cerevisiae } & \multicolumn{4}{|c|}{ T. cruzi } \\
\hline Prot & 3U5E & Length (aa) & Length (aa) & $\%$ ID & Prot MS & Ribo MS \\
\hline L9 & + & 191 & 189 & 46 & + & + \\
\hline L10 & + & 221 & 213 & 61 & + & + \\
\hline L11 & + & 174 & 192 & 69 & + & + \\
\hline L12 & - & 165 & 164 & 56 & + & + \\
\hline L13 & + & 199 & 218 & 39 & + & + \\
\hline L14 & + & 138 & 180 & 30 & + & + \\
\hline L15 & + & 204 & 204 & 57 & + & + \\
\hline L16 & + & 199 & 222 & 44 & + & + \\
\hline L17 & + & 184 & 166 & 54 & + & + \\
\hline L18 & + & 186 & 193 & 43 & + & + \\
\hline L19 & + & 189 & 357 & 50 & + & + \\
\hline L20 & + & 174 & 179 & 37 & + & + \\
\hline $\mathrm{L} 21$ & + & 160 & 159 & 41 & + & + \\
\hline L22 & + & 121 & 130 & 33 & - & + \\
\hline L23 & + & 137 & 139 & 69 & + & + \\
\hline L24 & + & 155 & 125 & 32 & + & + \\
\hline L25 & + & 142 & 226 & 45 & + & + \\
\hline L26 & + & 127 & 143 & 57 & + & + \\
\hline L27 & + & 136 & 133 & 43 & + & + \\
\hline L28 & + & 149 & 145 & 59 & + & + \\
\hline L29 & + & 59 & 71 & 82 & + & + \\
\hline L30 & + & 105 & 105 & 55 & + & + \\
\hline L31 & + & 113 & 188 & 42 & + & + \\
\hline L32 & + & 130 & 133 & 42 & + & + \\
\hline L33 & + & 107 & 149 & 42 & + & + \\
\hline L34 & + & 121 & 170 & 38 & + & + \\
\hline L35 & + & 120 & 127 & 44 & + & - \\
\hline L36 & + & 100 & 114 & 41 & + & + \\
\hline L37 & + & 88 & 84 & 57 & + & + \\
\hline L38 & + & 78 & 82 & 43 & - & + \\
\hline L39 & + & 51 & 51 & 60 & - & - \\
\hline L40 & + & 52 & 52 & 65.4 & + & - \\
\hline L41 & + & 25 & \multicolumn{4}{|c|}{ Not Found } \\
\hline L42 & + & 106 & 106 & 65 & - & + \\
\hline L43 & + & 92 & 90 & 61 & + & + \\
\hline
\end{tabular}

Table 1. Proteins from the large subunit. Left, S. cerevisiae: protein name, presence in the crystal structure (PDB: 3U5E) and number of residues. Right, T. cruzi homologues: amino acid length, percentage of identity and positive (+) or negative (-) detection by MS on whole parasites (Prot MS) or purified ribosomes (Ribo MS). 


\begin{tabular}{|c|c|c|c|c|c|c|}
\hline \multicolumn{3}{|c|}{ S. cerevisiae } & \multicolumn{4}{|c|}{ T. cruzi } \\
\hline Prot & $3 \mathrm{U} 5 \mathrm{C}$ & Length (aa) & Length (aa) & $\%$ ID & Prot MS & Ribo MS \\
\hline So & + & 252 & 245 & 52 & + & + \\
\hline S1 & + & 255 & 261 & 40 & + & + \\
\hline S2 & + & 254 & 263 & 58 & + & + \\
\hline S3 & + & 240 & 214 & 58 & + & + \\
\hline S4 & + & 261 & 273 & 50 & + & + \\
\hline S5 & + & 225 & 190 & 64 & + & + \\
\hline S6 & + & 236 & 250 & 49 & + & + \\
\hline S7 & + & 190 & 211 & 34 & + & + \\
\hline S8 & + & 200 & 221 & 47 & + & + \\
\hline S9 & + & 197 & 190 & 59 & + & + \\
\hline S10 & + & 105 & 161 & 34 & + & + \\
\hline S11 & + & 156 & 173 & 54 & + & + \\
\hline C12 & + & 143 & 142 & 30.8 & + & + \\
\hline 512 & 1 & 170 & 141 & 34.5 & + & - \\
\hline S13 & + & 151 & 151 & 62 & - & + \\
\hline S14 & + & 137 & 144 & 74 & + & + \\
\hline S15 & + & 142 & 152 & 53 & + & + \\
\hline S16 & + & 143 & 149 & 56 & + & + \\
\hline S17 & + & 136 & 141 & 57 & + & + \\
\hline S18 & + & 146 & 153 & 58 & + & + \\
\hline S19 & + & 144 & 167 & 35 & + & + \\
\hline S20 & + & 121 & 117 & 41 & + & + \\
\hline S21 & + & 87 & 251 & 43 & + & + \\
\hline $\mathrm{S} 22$ & + & 130 & 130 & 72 & + & - \\
\hline $\mathrm{S} 23$ & + & 145 & 143 & 68 & + & + \\
\hline S24 & + & 135 & 137 & 47 & + & + \\
\hline S25 & + & 108 & 110 & 39 & + & - \\
\hline S26 & + & 119 & 112 & 42 & + & + \\
\hline S27 & + & 82 & 86 & 61 & + & + \\
\hline S28 & + & 67 & 91 & 68 & - & + \\
\hline S29 & + & 56 & 57 & 54 & + & + \\
\hline S30 & + & 63 & 65 & 65 & - & - \\
\hline S31 & + & 152 & 150 & 60 & + & + \\
\hline RACK1 & + & 319 & 317 & 43 & + & + \\
\hline
\end{tabular}

Table 2. Proteins from the small subunit. Left, S. cerevisiae: protein name, presence in the crystal structure (PDB: 3U5C) and number of residues. Right, T. cruzi homologues: amino acid length, percentage of identity and positive (+) or negative (-) detection by MS on whole parasites (Prot MS) or purified ribosomes (Ribo MS). 
This analysis showed that T. cruzi ribosomal proteins are, in average, longer than the corresponding $S$. cerevisiae proteins. The extra regions in $T$. cruzi ribosomal proteins are generally at the $\mathrm{N}$ - or C-terminal ends. The most intriguing examples of these terminal extensions, when comparing to yeast, are TcL19 and TcS21 (blue rows on Tables 1 and 2, respectively), showing C-terminal extensions of 168 and 164 amino acids, respectively. These extensions are only present in kinetoplastids, although their length varies among species. MS analyses of T. cruzi ribosomes confirmed the presence of peptides matching to TcL19 and TcS21, strongly suggesting that these genes correspond to the functional ribosomal components [6]. The possible functional roles of these extensions, as well as the molecular mechanisms that generated them over time, constitute interesting fields for future studies.

It is interesting to note that S. cerevisiae L19 protein has been described as forming part of the polypeptide chain exit channel [8]. In addition to L19, the polypeptide chain exit channel is formed by L17, L25, L26, L31 and L35. All of these proteins show important extensions in T. cruzi, ranging from 41 amino acids (L35) up to 57 amino acids (L26). This fact can be related to the absence of a flat surface on this region in T. cruzi $80 \mathrm{~S}$ ribosome, in contrast to the corresponding region in the yeast ribosome [1].

Two putative homologue genes for the $\mathrm{S} 12$ protein sharing $65 \%$ of amino acids identity are present in the T. cruzi genome (named TcS12A and TcS12B). TcS12A is slightly closer to yeast $\mathrm{S} 12$ (34.5\% of amino acids identity) than TcS12B (30.8\% of amino acids identity). Both genes were expressed at the protein level [7] but only TcS12B was detected in the proteomic analysis of purified ribosomes (Table 2). Interestingly, there are also two genes in T. brucei (S12A and S12B) but only one in L. major, suggesting a gene duplication event after the divergence of Leishmania spp into the trypanosomatid lineage.

In other eukaryotes, such as mammals and yeast, ribosomal proteins S31 and L40 are synthesized as a C-terminal fusion with ubiquitin. Data mining revealed similar fusion genes in the T. cruzi genome. From these ubiquitin fusion proteins, only TcS31 (also named S27A) was detected by mass spectrometry on pure ribosomes.

Out of the 32 proteins found by sequence identity to S. cerevisiae 40 S proteins, 29 were detected by MS of T. cruzi ribosomes, including S13 and S28, which had not been detected in the proteome of T. cruzi [7]. Nevertheless, peptides matching to S22, S25 and S30 were not detected in the MS analysis of pure ribosomes. Interestingly, S30 was also not detected in MS studies on total extracts of T. cruzi (red row Table 2) [7].

For the large subunit (60S), out of the 48 yeast proteins screened, 47 were found to have a homologous gene in T. cruzi. The exception was L41, a short peptide of 25 amino acids long. The ribosome MS analysis detected all predicted proteins, excepting L1, L35, L39 and L40. From these, L1 and L35 were previously detected in epimastigote crude extracts [7]. Moreover, ribosome MS analysis detected two large subunit proteins that were not previously detected in the T. cruzi total proteome; L22 and L42 (Table 1). 
In addition to the previously discussed large and small subunit proteins ( $\mathrm{S}$ and $\mathrm{L}$ ), MS analyses detected other well-known ribosome components. Here we discuss some examples:

i. RACK1, a protein tightly associated to the small ribosomal subunit in eukaryotes, and apparently involved in the regulation of translation initiation $[9,10]$. This protein, present on the cryo-EM map of yeast ribosome was not detected on the cryo-EM map of T. cruzi (Figure 3). This difference can be explained by a weaker interaction between RACK1 and the ribosome in T. cruzi, compared to other species. This could result in too low amounts of RACK1 in purified ribosomes for cryo-EM visualization, but sufficient for MS detection.

ii. The ribosomal P proteins, a pentameric complex that form a long and protruding stalk on the large subunit involved in the translocation of the ribosome during the elongation step of protein synthesis. This complex is generally absent in cryo-EM and has not yet completely elucidated by crystallography due to its high flexibility (Figure 3). All proteins that form the $\mathrm{P}$ complex in $\mathrm{T}$. cruzi were detected in purified ribosome particles, indicating the presence of a functional pentameric complex. A complete description of $T$. cruzi stalk region, components, interactions and complex formation will be discussed below.

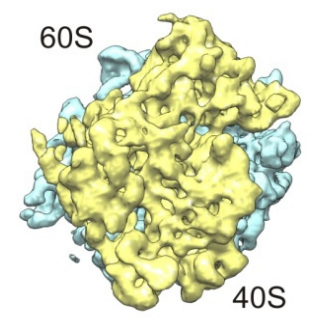

T. cruzi

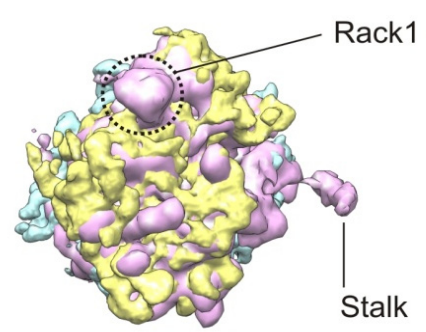

Stalk

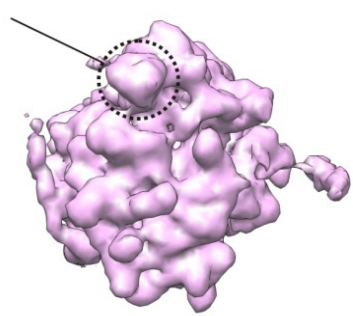

S. cerevisiae

Figure 3. Left, Cryo-EM of the T. cruzi $80 S$ ribosome. Center, superposition of T. cruzi and S. cerevisiae 80 S particles. Right, Cryo-EM of the S. cereviseae $80 S$ ribosome. Rack 1 and the stalk region, which are only present in S.cerevisiae ribosome are indicated.

\subsection{The ribosomal stalk: variable components and assembly}

The large subunit of the eukaryotic ribosome possesses a long and protruding stalk formed by the ribosomal $\mathrm{P}$ proteins. This structure is involved in the translocation of the ribosome during the elongation step of protein synthesis through interaction with the elongation factor 2 (EF-2) [11]. Although the elongation step is a highly conserved process, the stalk is one of the most variable regions of the ribosome. The proteins forming this structure in prokaryotes and eukaryotes show very low sequence similarity. Moreover, among eukaryotes, the composition of the stalk is also variable, due to gene duplications and sequence divergence of genes encoding $\mathrm{P}$ proteins. In general terms the eukaryote complex is formed by the ribosomal $\mathrm{P}$ proteins, including $\mathrm{P0}$ (a $34 \mathrm{kDa}$ polypeptide) as a central component of the stalk and two distinct but closely related proteins of about $11 \mathrm{kDa}, \mathrm{P} 1$ and $\mathrm{P} 2$. The number of ribosomal P1/P2 proteins varies among species and these variations have 
consequences in the stalk composition. In mammals, the P1 and P2 families have only one member and the stalk is formed by two identical copies of each P1 and P2 proteins, linked to P0 [12]. The binding of P2 protein to P0 can only be detected in the presence of P1, suggesting a pivotal role of the latter in the conformation of the stalk [13]. In S. cerevisiae there are two P1 $(\alpha$ and $\beta)$ and two P2 ( $\alpha$ and $\beta$ ) proteins [14] and the stalk seems to be organized in preferential pairs; $\mathrm{P} 1 \alpha / \mathrm{P} 2 \beta$ and $\mathrm{P} 1 \beta / \mathrm{P} 2 \alpha$. Again, both $\mathrm{P} 1$ proteins seem to be necessary for the binding of the corresponding P2 partners to P0 [15]. In T. cruzi, five components of the stalk have been identified: P0, of approximately $34 \mathrm{kDa}$, containing a Cterminal end that deviates from the eukaryotic $\mathrm{P}$ consensus and bears similarity with Archaea L10 protein; and four proteins of about $11 \mathrm{kDa}(\mathrm{P} 1 \alpha, \mathrm{P} 1 \beta, \mathrm{P} 2 \alpha$ and $\mathrm{P} 2 \beta)$ with the typical eukaryotic P consensus sequence at their C-terminal end [16, 17]. It should be noted that independent gene duplication events have originated the P1 and P2 subtypes in yeast and trypanosomatids.

Combining yeast two-hybrid technique and surface plasmon resonance (SPR) it was possible to make a complete interaction map of T. cruzi ribosomal P proteins [17-19]. These two techniques were both necessary to fully characterize the complex and to map the interaction regions in P0 (Figure 5). This analysis exposed some trypanosomatid-specific features among $\mathrm{P}$ proteins interactions. TcP0 protein (the central component of the stalk) was able to interact with all $\mathrm{P} 1 / \mathrm{P} 2$ proteins. Both $\mathrm{P} 1$ proteins were unable to interact with each other nor to homo-oligomerize. Interestingly, both $\mathrm{P} 2$ proteins showed a highly redundant interaction profile but P2 $\alpha$ was not able to interact with P1 $\alpha$. Therefore, if we focus in a T. cruzi stalk composed of five different ribosomal $\mathrm{P}$ proteins the only possible arrangement for the low molecular weight protein association will be: $\mathrm{P} 1 \alpha-\mathrm{P} 2 \beta / \mathrm{P} 1 \beta-\mathrm{P} 2 \alpha$. Any other possible combination will exclude one of the four components. This association pattern resembles that observed in yeast; although not completely, because of the presence of highly interacting components like P2 $\beta$. Consequently, it is possible to postulate heterogeneity on the stalk composition in T. cruzi, due to redundancy of some of its components.

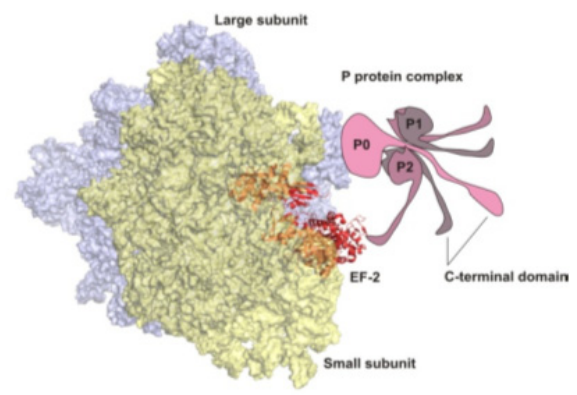

Figure 4. Surface image of yeast $80 \mathrm{~S}$ ribosome crystallography in complex with the EF- 2 (red). The postulated location of the ribosomal $\mathrm{P}$ proteins on the stalk region of the large subunit is illustrated.

Since all four small $\mathrm{P}$ proteins can bind to P0 the question was whether TcP0 can simultaneously bind two or four proteins. Despite the accumulated data about stalk organization in several model organisms, the interaction among P0 and P1/P2 proteins is not 
completely understood at the molecular level. Tsurugi and Mitsui [20], based on sequence analysis of $S$. cerevisiae and $H$. sapiens $\mathrm{P} 0$, proposed the presence of eight putative hydrophobic zippers involved in the interaction with $\mathrm{P} 1$ and $\mathrm{P} 2$ proteins. Functional complementation assays in yeast using C-terminal truncated $\mathrm{P} 0$ variants showed that deletion of 87 amino acids (the entire putative hydrophobic zipper) abolishes the binding of P1/P2 proteins to the ribosome [21]. Using yeast two-hybrid technique ( $\mathrm{Y} 2 \mathrm{H})$, it has been shown that the 100 amino acid long C-terminal domain of P0 strongly interacts with P1 and P2 proteins [22]. However, the last 50 amino acids of $\mathrm{P} 0$ (including only two of the eight hydrophobic residues) were not able to interact with $\mathrm{P} 1 / \mathrm{P} 2$. More recently, using $\mathrm{Y} 2 \mathrm{H}$ and deletion mutant strains, the P0 region between positions 213 and 260 has been involved in the interaction with the P1/P2 proteins in S. cerevisiae. In contrast, mutation of the putative interacting leucine residues in this region did not impair the binding of P1 and P2 proteins [23]. Based on the crystal structure of the archaeon Pyrococcus horikoshii stalk complex recently reported [24], it was possible to identify two putative P1/P2 interaction sites in TcP0. The first site is situated between aminoacids 222 and 232 and the second between aminoacids 248 and 258. Although the general mechanism mediating the interaction between $\mathrm{P} 0$ and $\mathrm{P} 1 / \mathrm{P} 2$ proteins seems to be similar among all species, it should be noted that some of the residues on $\mathrm{TcP} 0$ involved in this interaction are not strictly conserved. This result is in agreement with other studies showing that ribosome from yeast strains carrying heterologous P0 proteins are not able to bind $S$. cerevisiae P1/P2 proteins efficiently $[25,26]$. Altogether our data indicate that TcP0 possesses two $\mathrm{P} 1 / \mathrm{P} 2$ interaction sites and that $\mathrm{P} 1 / \mathrm{P} 2$ proteins can associate in pairs (P1 $\alpha-\mathrm{P} 2 \beta / \mathrm{P} 1 \beta-\mathrm{P} 2 \alpha)$ but it was not known whether a hierarchy for P1/P2 association to TcP0 exists. To answer this question we performed a sequential SPR analysis in which we randomly injected one protein after the other (P1/P2) without regeneration steps on a sensor chip containing TcP0 [19]. This study showed that it is possible to form stable pentameric complexes when any of both P1 proteins were first injected. There were a few other combinations that raised stable complexes but in general terms it is possible to conclude that the injection of multi-interacting proteins (like P2 $\beta$ and to a lesser extent $\mathrm{P} 2 \alpha$ ) at the beginning blocks the binding of the other components of the complex. This also means that other complexes containing not all P1/P2 proteins are possible. Unfortunately, there are no functional data available.

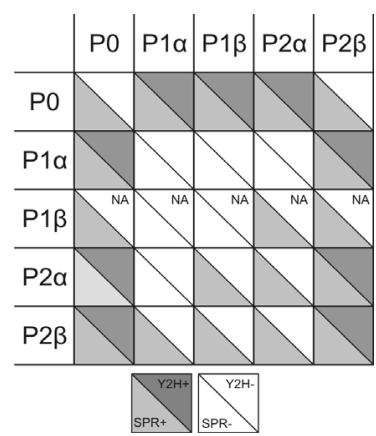

Figure 5. Summary of $\mathrm{P}$ protein interactions assessed by SPR and yeast two hybrid technique $(\mathrm{Y} 2 \mathrm{H})$. NA: Not analyzed. 
Finally, the complete picture of the system can be illustrated in Figure 6, where the T. cruzi stalk resembles that of yeast due to the ability of all $\mathrm{P} 1 / \mathrm{P} 2$ proteins to interact with $\mathrm{P} 0$.
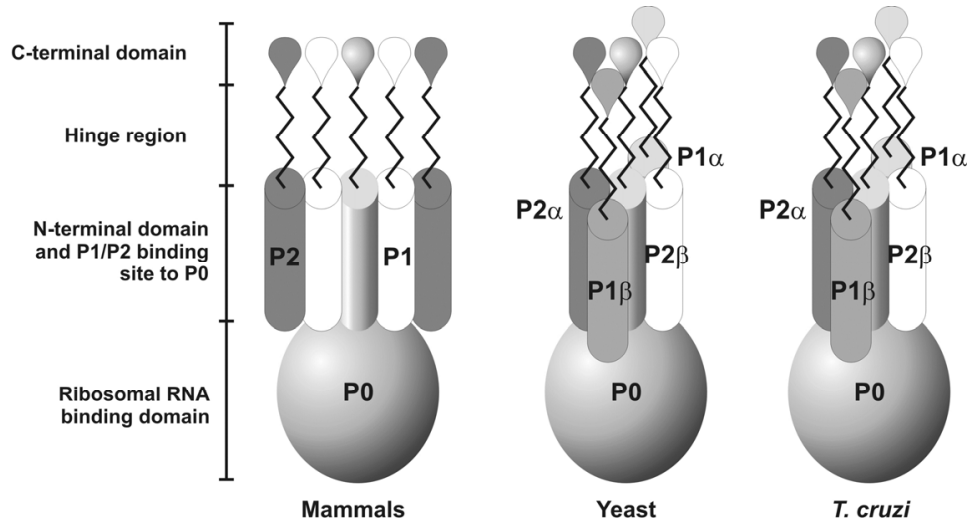

Figure 6. Assembly of the P complex in mammals, yeast and the proposed model for T.cruzi complex.

As it was mentioned before, the C-terminal end of ribosomal P proteins interacts with EF-2 and this interaction is essential during the elongation step of protein synthesis. Notably, antibodies against the C-terminal end of T. cruzi ribosomal P proteins are present in the sera of a high percentage of chronic chagasic patients. These antibodies are specific for the $T$. cruzi C-terminal peptide of ribosomal $\mathrm{P}$ proteins, being unable to recognize the mammalian epitope. This specificity is due to only one amino acid change (Ser by Glu) [27-30]. In a previous work, we have obtained a recombinant single chain antibody (scFvC5), derived from a monoclonal antibody against the C-terminal region of T. cruzi P2 $\beta$ protein $[31,32]$. This recombinant antibody, similarly to human antibodies from chagasic patients, shows very high selectivity toward the parasite epitope. In Western blot assays, scFvC5 specifically recognized $\mathrm{P}$ proteins on extracts of trypanosomatids T. cruzi. T. brucei and Crithidia fascilculata, but it did not detect their rat counterparts. Based on earlier reports showing that antibodies (and their recombinant single chain versions) directed against the mammalian Cterminal end of $\mathrm{P}$ proteins inhibit protein synthesis in cell-free systems [33], we reasoned that $\mathrm{scFvC5}$ would selectively block translation process by trypanosomatid ribosomes. As expected, scFvC5 strongly inhibited the incorporation of radioactive amino acids when trypanosomatid ribosomes (from T. cruzi, T. brucei and C. fasciculata), but not mammalian (Rattus norvegicus) ribosomes were used under identical experimental conditions [30]. Moreover, the translation inhibition on trypanosomatid ribosomes could be reverted by preincubation of the scFvC5 with the peptide corresponding to the T. cruzi epitope, but not with the mammalian equivalent. Therefore, we evaluated the ability of this recombinant antibody to inhibit protein synthesis in vivo, by using a tightly regulated inducible expression vector in T. brucei. The growth of parasites was significantly delayed when the scFvC5 expression was induced; clearly showing that blocking of the C-terminal end can be used as a strategy to inhibit trypanosomatid protein synthesis in vivo. In addition, and taking into account that the crystal structure of the monoclonal antibody originating scFvC5 has been reported [34] 
(PDB 3SGE), the antibody combining site would be an interesting starting point for designing peptide mimetics as specific inhibitors of trypanosomatid translation.

\section{Functional analysis}

\subsection{Translation activity: Initiation and elongation factors}

\subsubsection{Trans-splicing of trypanosomatid mRNAs}

In several organisms, a variable proportion of mRNAs are processed by a mechanism named spliced-leader (SL) trans-splicing, which transfers a short RNA sequence (the SL) from the $5^{\prime}$ end of a specialized non-mRNA molecule, the SL RNA, to unpaired spliceacceptor sites on pre-mRNA molecules. As a result, depending on the organism, a variable proportion of the mRNAs acquires a common 5' sequence. The SL trans-splicing mechanism is widely and patchily distributed across phylogenetically distant organisms. The evolutionary origin of this process is still an enigma, and two different hypotheses have been postulated [35]:

i. SL trans-splicing was present in an ancestral eukaryotic organism and has been lost in many different lineages, or

ii. SL trans-splicing appeared independently several times during evolution of eukaryotes.

Although this point has not been solved yet, recent evidences from analysis of large ESTs and genomic databases, seem to better support the second hypothesis [36, 37].

Several different functions for the SL sequences have been reported [35], among them:

i. providing a 5' cap structure for protein coding RNAs transcribed by RNA polymerase I

ii. Converting polycistronic transcripts into capped, monocistronic mRNAs

iii. enhancing mRNA translational efficiency

In trypanosomatids, $100 \%$ of their mRNA is processed by SL trans-splicing, adding a $39 \mathrm{nt}$ sequence. Besides the universally conserved 7-methyl guanosine cap, which is linked to the first nucleotide via a $5^{\prime}-5^{\prime}$ triphosphate bridge, the first four nucleotides of the SL are all methylated at the ribose ring. In addition, the first $\left(\mathrm{m}_{2}{ }^{6} \mathrm{~A}\right)$ and fourth $\left(\mathrm{m}^{3} \mathrm{U}\right)$ nucleotides are methylated at the base [38] (Figure 7). This unusually modified structure, known as the cap4 , is the most highly modified cap structure of all eukaryotic cells. Due to the important role of the mRNA 5' end during eukaryotic translation initiation, a role for the SL structure has been proposed in the process. By using cell lines of Leishmania tarentolae expressing modified SL sequences, it has been shown that these modifications do not affect transcription nor trans-splicing efficiency. In contrast, mutations of the SL region spanning nucleotides 10-29 decreased the methylation extent and polysome association of mRNAs, demonstrating a direct role for the cap4 methylations and/or the primary sequence of the SL in the translation process [39]. More recently, Zamudio et al used Trypanosoma brucei strains lacking one or more of the three 2'-O-ribose methyl transferases involved in the cap4 biogenesis, to specifically evaluate the role of SL methylation in the absence of sequence changes [40]. In 
this study, attempts to derive cells with complete loss of mRNA cap ribose methylation were unsuccessful, indicating an essential role in kinetoplastid biology. Moreover, even when cells lacking the kinetoplastid-specific ribose methylation at positions 3 and 4 were viable, they showed a decreased rate of protein synthesis, clearly showing a role for these modification (in the absence of SL sequence changes) in the translation process. The above mentioned evidences, demonstrating a direct role of hypermethylated SL in trypanosomatid protein synthesis, reinforce the hypothesis that translation initiation would show unique features in these organisms.

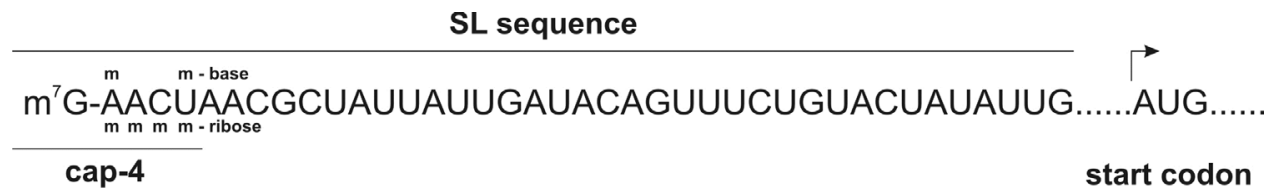

Figure 7. The T. cruzi spliced leader sequence

\subsubsection{Initiation factors}

Cap-dependent initiation in eukaryotes is a very complex, highly regulated limiting step of translation. In this process, the $5^{\prime}$ cap interacts with a multi-protein complex named eIF4F, formed by at least three proteins: eIF4A, an ATP-dependent RNA helicase; eIF4E, the capbinding protein; and eIF4G, a scaffold protein that interacts with the poly(A) binding protein, IF4A and IF4E. Data mining on the Leishmania major genome database has revealed four eIF4Es (LmEIF4E1-4), two eIF4As (LmEIF4A1-2) and five eIF4Gs (LmEIF4G1-5) putative proteins [41].

The presence of multiple homologues for the different IF4F subunits in trypanosomatids, and in some cases in other eukaryotes, makes it difficult to identify these proteins that are actually involved in translation. In addition, knowledge of the protein synthesis in trypanosomatids is inferred by indirect evidences such as sequence similarities. Moreover, proteins with high homology to translation initiation factors are involved in other processes such as mRNA processing. Since orthologous proteins have, in general, conserved functional roles, phylogeny analysis could have some clues for identifying those proteins involved in protein synthesis. Below we discuss the biochemical, molecular and phylogenetic evidences available on the IF4F components.

\subsubsection{Trypanosomatid eIF4E}

A recent phylogenetic analysis of IF4E-family members has revealed that many organisms contain multiple genes encoding proteins with sequence similarity to prototypical IF4E proteins [42]. Unfortunately, no trypanosomatid IF4E-family members were included in this analysis.

The highly modified cap of trypanosomatids suggests that eIF4E orthologous in these organisms would show atypical features. The L. major genome revealed four putative eIF4E encoding genes (named LeishIF4E-1 to -4) with limited homology. All of them have easily 
identifiable orthologues in T. brucei and T. cruzi, suggesting that their functional roles are conserved among trypanosomatids, as can be inferred from phylogenetic analysis (Figure 8). In contrast, the phylogenetic relationships between putative trypanosomadid IF4E proteins and the homologous translation factors characterized in other species seem to be much more complex. This complex evolutionary scenario strongly suggests that multiple gene duplications have taken place during evolution of IF4E homologous genes, some of them before, and others after the evolutionary divergence between organisms, yielding both paralogous and orthologous genes. In spite of this complexity, it is clear that trypanosomatid IF4E-2 and IF4E-3 are paralogous genes originated by duplications into the trypanosomatid lineage.

The L. major genes have been cloned and their respective protein products have been biochemically characterized [43]. The corresponding recombinant proteins showed variable relative affinities for chemically synthesized mammalian and trypanosomatid cap structures. Despite a detailed study of these proteins, including pull-down assays with a mammalian interacting partner of eIF4E, analysis of co-sedimentation with polysome fractions and detection at different stages of the parasite, no clear conclusions could be obtained leading to an eIF4E candidate. Moreover, none of these proteins was able to rescue the phenotype of a $S$. cerevisiae null strain, indicating a significant functional divergence of these proteins in the trypanosomatid lineage. This conclusion is reinforced by the observation that several eIF4E homologues from phylogenetically distant organisms (mammals, Drosophila melanogaster, zebrafish and Arabidopsis) are functional in yeast (red letters in Figure 8) [44-47]. Interestingly, other IF4E homologous from these species are not functional in yeast, suggesting that they are involved in roles other than translation initiation (i.e. translation repression, binding to nuclear mRNAs).

More recently, the orthologue genes from T. brucei (TbEIF4E-1 through 4) have been functionally analyzed in vivo [50]. By using RNAi knock down of different TbIF4E genes, combined with metabolic labeling with radioactive amino acids, this study strongly suggested that TbIF4E-3 (and also probably TbIF4E-4) are directly involved in protein synthesis. This is supported by their cytosolic localization, in contrast to the TbIF4E- 1 and 2 proteins, which are both nuclear and cytosolic. Unfortunately, no yeast complementation assays were performed with T. brucei proteins which could be correlated with the data of their L. major orthologues.

A similar situation seems to take place in the ancient eukaryotic, non-kinetoplastid, Giardia lamblia. Two IF4E homologous proteins have been identified and characterized; Gl_IF4E-1 and Gl_IF4E-2. Notably, Gl_IF4E-1 seems to be the orthologous gene of LmIF4E-4, whereas Gl_IF4E-2 is more closely related to LmIF4E-1. This suggests that duplications of genes encoding IF4E-family proteins have taken place very early during eukaryotic evolution. Functional analyses suggest that Gl_IF4E-2 is involved in protein synthesis. However, similarly to the Leishmania proteins, none of these IF4E homologues is able to rescue a null phenotype in yeast [51]. These observations suggest that early eukaryotes, such as Giardia and trypanosomatids, have major functional differences with model organisms in protein synthesis initiation, particularly in the case of IF4E. Moreover, it seems that after divergence 


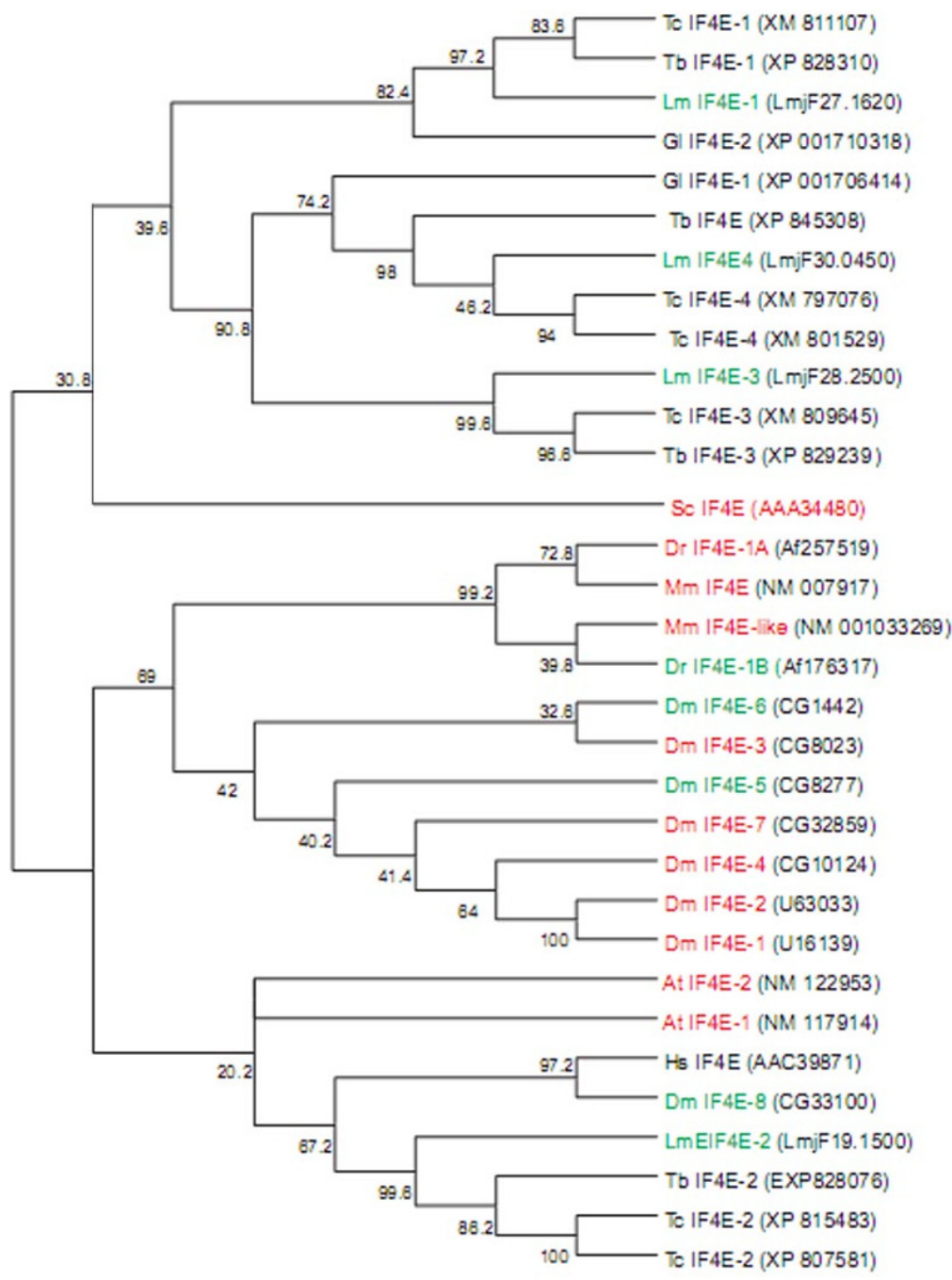

Figure 8. Inferred phylogenetic relationships among putative trypanosomatid IF4E proteins and homologous proteins from other eukaryotes. Sequences are derived from $H$. sapiens (Hs), Mus musculus $(\mathrm{Mm})$, S. cerevisiae (Sc), Drosophila melanogaster (Dm), Giardia lamblia, $(\mathrm{Gl})$, Arabidopsis thaliana (At), Danio rerio (zebra fish) (Dr), L. major (Lm), T. brucei (Tb) and T. cruzi (Tc). Accession codes for GenBank or $L$. major database (http://www.genedb.org/Homepage/Lmajor) are shown in parenthesis. Sequences covering the IF4E domain (PFAM 01652) were aligned using T-COFEE under default parameters (http://tcoffee.vital-it.ch). LG+G was chosen as the best evolutionary model using ProtTest [48]. PhyML was run using the algorithm Subtree Pruning and Regrafting (SPR) [49] with 5 initial starting trees. To estimate the robustness of the phylogenetic inference, 500 bootstrap replicates were run. Color letters indicate whether these proteins are able (red) or unable (green) to complement a S. cerevisiae deficient strain. Arrows indicate the proteins that have proved functional roles in protein synthesis in ancient eukaryotes (trypanosomatid and Giardia). 
of Giardia and trypanosomatid lineages, different paralogous genes acquired a major functional role in protein synthesis initiation, giving an additional level of complexity to the evolution of IF4E-family genes. Data from studies in L. major and T. brucei suggest that functional roles can be inferred by orthology into the trypanosomatid lineage, but these conclusions cannot be further extrapolated to other eukaryotic organisms.

\subsubsection{Trypanosomatid IF4A}

The sequence analysis of IF4A gives a more simple interpretation than IF4E, because of a lower number of homologous genes in each species, and the fact that these proteins show a higher degree of sequence conservation. IF4A belongs to a DEAD-box helicase family, which includes translation factors, as well as proteins involved in splicing. The inferred phylogenetic relationships amongst these proteins yield two clearly separate clades (Figure 9). In each of these clades, the phylogeny of the proteins is coincident with that of their organisms, strongly suggesting that a unique duplication event of an ancestor IF4A gene took place before the divergence of early eukaryotes. As can be seen, S. cerevisiae IF4AI (P10081.3) and Homo sapiens IF4A2 (Q14240.2), both having demonstrated roles in protein synthesis initiation are in the same clade. Consistently, the paralogous proteins IF4A-like FAL1 (Q12099.1) and IF4AIII (P38919.4), from S. cerevisiae and H. sapiens, respectively, have been shown to be involved in splicing, and consistently they belong to a separate clade. This analysis suggests that trypanosomatid IF4A homologous proteins of the first clade, would be expected to be involved in protein synthesis initiation.

The first functional analysis of a trypanosomatid IF4A homologue was performed with a Leishmania infantum protein (LeIF; XP_001462692.1), initially identified as an antigen inducing IL12 mediated immune response in infected patients [52]. This protein shows RNA-dependent ATPase and ATP-dependent RNA helicase activities and interaction with yeast eIF4G in vitro. However, LiIF4A does not complement a yeast eIF4A deficient strain, leading the authors to suggest that LiIF4A is indeed the orthologue of human eIF4AIII, involved in splicing. The phylogenetic analysis (Figure 9) allows us to propose an alternative hypothesis, since LiIF4A is located in the translation factor clade. We propose that this protein is indeed an initiation translation factor and that its inability to complement yeast cells reflects the evolutionary functional divergence of trypanosomatid protein synthesis. This hypothesis is supported by the finding of another gene in the genome of $L$. infantum; LiIF4A-like (XP_001470194.1), which groups with the clade of splicing factors. Additional evidences supporting this hypothesis have been provided by knock-down of the two T. brucei paralogous genes, combined with metabolic labeling with radioactive amino acids in silenced strains. These studies showed that knock-down of TbIF4AI, but not of TbIF4AIII, specifically decreases the rate of protein synthesis [53]. Figure 9 shows a phylogenetic tree of IF4A homologous proteins from different organisms, highlighting those with demonstrated roles in translation (blue letters) or other functions than translation (pink letters). 


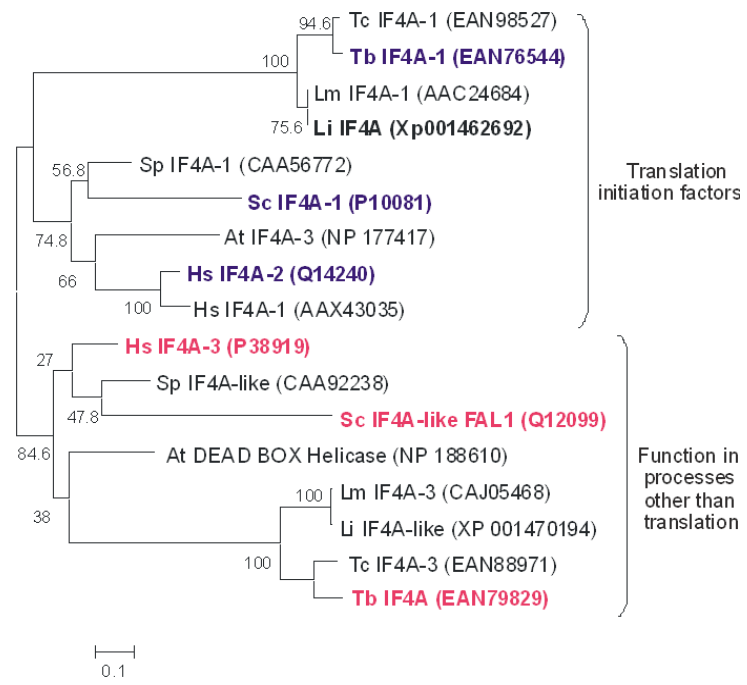

Figure 9. Inferred phylogenetic relationships among putative trypanosomatid IF4A proteins and homologous proteins from other eukaryotes. The complete open reading frames were aligned using TCOFEE under default parameters. LG+G was chosen as the best evolutionary model. All those proteins with demonstrated role in protein synthesis are located in one of these clades, whereas the second clade seems to group the proteins involved in splicing. ScIF4AI (P10081.3) [54] and HsIF4A2 (Q14240.2) [55] (blue letters), have demonstrated roles in protein synthesis, whereas ScIF4A-like FAL1 (Q12099.1) [56] and HsIF4AIII (P38919.4) [57] (pink letters), have been implicated in other processes. L. infantum LiIF4A (XP_001462692.1) (bold and underlined) has been cloned and characterized as unable to complement an IF4A yeast null strain [52].

\subsubsection{Trypanosomatid IF4G}

As mentioned before, IF4G is a scaffold protein that coordinates the assembly of the translation initiation factors and the $40 \mathrm{~S}$ ribosomal subunit. The middle domain of IF4G (MIF4G) is the hallmark of these proteins. In addition, this domain is also present in several other proteins not involved in translation, such as the nuclear cap-binding protein CBP80. This fact reflects the common origin shared by IF4G with other cap-binding proteins, being difficult to deduce, based only on homology, which proteins harboring this domain have indeed initiation factor activity.

Five different proteins harboring MIF4G domain are present in the genome of L. major, named LmIF4G-1 to LmIF4G-5 [41]. All of them have clearly predictable orthologues in $T$. brucei and T. cruzi. From these, and based on different functional assays including pulldown, yeast-two hybrid and polysome profiles, LmIF4G-3 seems to be the most probable orthologous with a major role in translation initiation [41, 58]. Interestingly, phylogeny inference shows that trypanosomatid IF4G proteins form a separate clade, implying several gene duplication events into the trypanosomatid lineage (Figure 10). It has been postulated that MIF4G domain appeared during the early stages of eukaryotic evolution [59]. 
Interestingly, TBLASTN searches on Giardia genome database using many different MIF4G domains as probe, give no significant hits. This strongly suggests that this domain appeared during the evolution of eukaryotes, after the divergence of the Giardia lineage. This implies that trypanosomatids would be amongst the earliest diverging organisms harboring IF4G proteins.

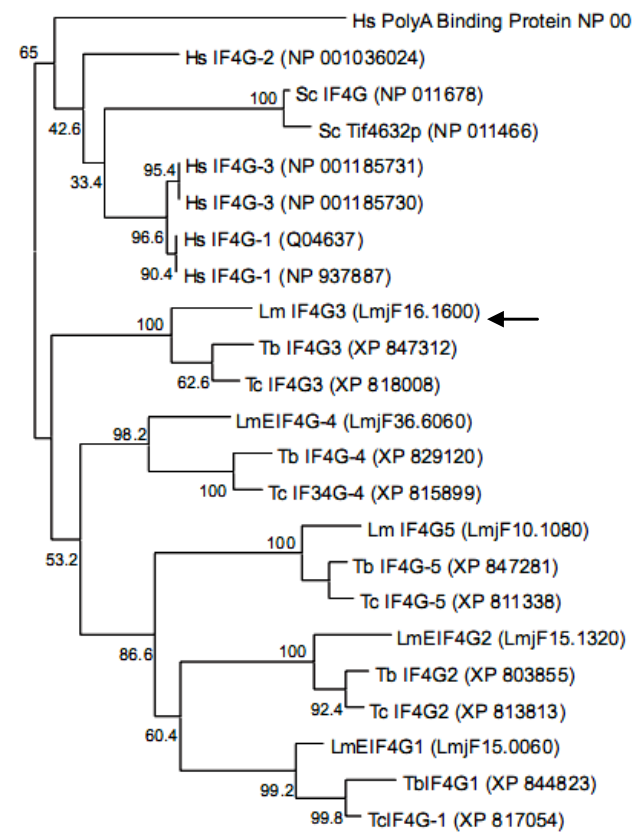

Figure 10. Inferred phylogenetic relationships among putative trypanosomatid IF4G and homologous proteins from other eukaryotes. Sequences are derived from Homo sapiens (Hs), Saccharomyces cerevisiae (Sc), L. major (Lm), T. brucei (Tb) and T. cruzi (Tc). Accession codes for GenBank or L. major database (http://www.genedb.org/Homepage/Lmajor) are shown in parenthesis. Sequences covering the MIF4G domain (PFAM cd11559) were aligned using T-COFEE under default parameters (http://tcoffee.vitalit.ch). $L G+G$ was chosen as the best evolutionary model using ProtTest [48]. PhyML was run using the algorithm Subtree Pruning and Regrafting (SPR) [49] with 5 initial starting trees. To estimate the robustness of the phylogenetic inference, 500 bootstrap replicates were run. LmIF4G-3, the only trypanosomatid IF4G-like protein that has been experimentally associated to the formation of the IF4F complex is indicated by an arrow.

\subsubsection{Elongation factors}

The elongation step of protein synthesis has been highly conserved trough evolution, in comparison with initiation. According to this, the sequences encoding for elongation factors can be easily inferred by homology. However, elongation factors from bacteria and eukaryotes are not functionally interchangeable. Moreover, the specificity of ribosomes for their homologous elongation factors can be changed by interchanging their stalk components [60]. 
The eukaryotic EF-2 is a GTPase involved in translocation of the peptidyl-tRNA from the A site to the $\mathrm{P}$ site on the ribosome. Two major mechanisms involving EF-2 regulate protein elongation. One of them is EF-2 reversible phosphorylation [61]. The second is EF-2 inactivation by ADP-ribosylation of a diphthamide residue (a post-translational modification of a conserved histidine residue) $[62,63]$. In addition, EF-2 interacts with the ribosomal $P$ proteins that form the stalk region [64]. A direct interaction between the stalk base and the EF-2 has been visualized by cryoelectron microscopy in yeast [65]. Real time measurements, using surface plasmon resonance (SPR), demonstrated the binding of rat EF-2 to rat P1 and P2 proteins, with a higher affinity for $\mathrm{P} 1\left(\mathrm{~K}_{\mathrm{D}} 3.810^{-8} \mathrm{M}\right)$ than for $\mathrm{P} 2\left(\mathrm{~K}_{\mathrm{D}} 2.210^{-6} \mathrm{M}\right)$ [66]. Moreover, antibodies against the conserved C-terminal region of ribosomal $\mathrm{P}$ proteins inhibited the elongation step of protein synthesis, blocking the binding of EF-2 to the ribosome as well as its ribosome dependent GTPase activity [67]. Below, we will summarize evidence of structural and functional divergences amongst eukaryotes at the ribosomal stalk level, and its partner EF-2.

It has been largely demonstrated that the antifungal sordarin, which selectively inhibits some eukaryotic ribosomes, acts at the elongation step. Detailed studies about its action mechanisms have revealed that specificity of this compound for a group of fungi is due to specific molecular features of their stalk components (mainly the P0 protein) and EF-2 [68, 69].

On the other hand, trypanosomatid ribosomes have been shown to be highly resistant, in comparison to mammalian particles, to two ribosome inactivating proteins; ricin and trichosanthin [70, 71]. These toxins bind initially to the ribosomal stalk and depurinate a conserved base on the $28 \mathrm{~S}$ rRNA, blocking the binding of EF-2 to ribosomes, supporting the idea that the trypanosomatid stalk has specific features.

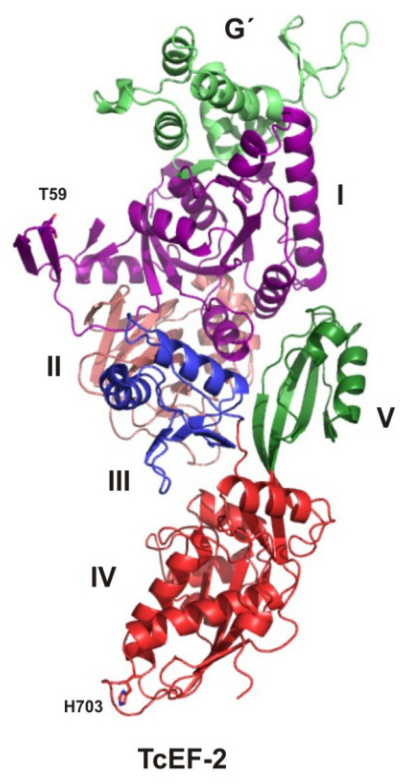

Figure 11. Model of T. cruzi EF-2. 
The amino acid sequence of TcEF-2 was identified by probing the T. cruzi Gene Data Base (http://www.genedb.org) with the amino acid sequence of $S$. cerevisiae EF-2. Two identical copies of the TcEF-2 gene were found. The TcEF-2 protein was 60\% identical to ScEF-2, and both EF-2 shared over $76 \%$ homology. In contrast to ScEF-2 that is regulated by phosphorylation of Threonine 57, TcEF-2 presented a Methionine in this position, suggesting a lack of regulation through phosphorylation (Figure 11). The histidine involved in ADP-ribosylation in ScEF-2 (H699) is conserved in trypanosomatids (H703 in T. cruzi), and consequently, diphtheria toxin inactivates protein synthesis in T. brucei [70]. Secondary structure comparative analysis showed an overall conserved architecture. The four canonical helices of domain I (GTPase) were conserved as well as the motif involved in nucleotide binding [72]. Using PHYRE protein fold recognition server [73], we generated a 3D model of TcEF-2 that contains the six structural domains described for ScEF-2 [74]. Therefore, taking into account the high structural similarity between ScEF-2 and TcEF-2, it may be assumed that the different domains have conserved similar functions. The interaction of TcEF-2 with the ribosomal $\mathrm{P}$ proteins showed that all $\mathrm{P} 1 / \mathrm{P} 2$ proteins interacted with TcEF-2 with similar affinities. Interestingly, TcP0 showed a decreased affinity in concordance with its modified C-terminal region. Our results are in agreement with those reported by Bargis-Surgey et al. [67] for the rat homologues. However, in T. cruzi no differential interaction between the EF-2 and the different P1/P2 proteins was observed.

Unfortunately, data from functional comparison between trypanosomatid EF-2 and its orthologous in model species, such as yeast functional complementation, are not yet available.

\subsection{Selective inhibition of trypanosomatid ribosomes by paromomycin}

Aminoglycosides are a group of antibiotics binding to the decoding center of rRNA. As a result, aminoglycosides interfere with protein synthesis, facilitating amino acid misincorporation. The target site of aminoglycosides is the helix 44 of the $18 \mathrm{~S}$ rRNA. As noted before, this helix is located in the ES12, the only expansion segment which is shorter in T. cruzi than in other eukaryotes, being intermediate between bacterial and higher eukaryotes [1]. Paromomycin is an aminoglycoside antibiotic with low toxicity to mammalian cells. This antibiotic has shown strong anti-leishmania activity when used alone or in combination with other drugs [75]. In a recent work, advances on the molecular basis for the differential effect of paromomycin on Leishmania have been reported [4]. In this work it was demonstrated that paromomycin selectively inhibits the rate of in vitro protein synthesis by trypanosomatid ribosomes, comparing to mammalian extracts. Moreover, the effect of paromomycin was even more dramatic when translation misreading was evaluated. Finally, affinity measurements using BIACORE demonstrated that paromomycin displays high affinity for the RNA oligonucleotide corresponding to the decoding site of trypanosomatids, whereas no interaction was detected with the oligonucleotide corresponding to the mammalian site.

\section{Conclusion}

In summary, we have described several direct and indirect evidences justifying future indeep studies on the trypanosomatid protein synthesis machinery. 
The sequence and functional analysis of trypanosomatid homologous to well-characterized initiation factors, along with the fact that SL sequence is present in all the trypanosomatid mRNAs, strongly suggest that protein synthesis initiation would have remarkable unique features in these protozoa. This hypothesis is reinforced by structural features of T. cruzi ribosomes, where a large, trypanosomatid-specific rRNA extra density is present adjacent to the platform region, involved in translation initiation.

The sequence analysis of ribosomal proteins also shows peculiarities in T. cruzi, being L19 and S21 proteins the most notable examples, possessing large trypanosomatid-specific domains with unknown functional roles.

Even when the elongation step of translation is much more conserved through evolution, the stalk composition and structure also seem to have special features in trypanosomatids. Consistently with this observation, some ribotoxins binding to this site show differential selectivity against parasite ribosomes. Unfortunately, no functional data are available on the functional similarities between trypanosomatid and mammalian elongation factors.

Paromomycin is the first example of a compound with a well characterized action mechanism, showing higher activity on trypanosomatid translation machinery in comparison with its mammalian counterpart. This fact, along with the number of key features of trypanosomatid ribosomes and translation factors, should strongly encourage the future search for novel, trypanosomatid-specific translation inhibitors.

\section{Author details}

Maximiliano Juri Ayub and Walter J. Lapadula

IMIBIO-SL, CONICET, Universidad Nacional de San Luis, Argentina

Johan Hoebeke

UPR9021 of the C.N.R.S. "Immunologie et Chimie Thérapeutiques", France

Cristian R. Smulski

Department of Biochemistry, University of Lausanne, Switzerland, UPR9021 of the C.N.R.S. "Immunologie et Chimie Thérapeutiques", France

\section{References}

[1] Gao H, Ayub MJ, Levin MJ, Frank J. The structure of the $80 S$ ribosome from Trypanosoma cruzi reveals unique rRNA components. Proc Natl Acad Sci U S A. 2005 Jul 19;102(29):10206-11.

[2] Frank J. Toward an understanding of the structural basis of translation. Genome Biol. 2003;4(12):237.

[3] Wuyts J, De Rijk P, Van de Peer Y, Pison G, Rousseeuw P, De Wachter R. Comparative analysis of more than 3000 sequences reveals the existence of two pseudoknots in area 
V4 of eukaryotic small subunit ribosomal RNA. Nucleic Acids Res. 2000 Dec 1;28(23):4698-708.

[4] Fernandez MM, Malchiodi EL, Algranati ID. Differential effects of paromomycin on ribosomes of Leishmania mexicana and mammalian cells. Antimicrob Agents Chemother. Jan;55(1):86-93.

[5] Spahn CM, Jan E, Mulder A, Grassucci RA, Sarnow P, Frank J. Cryo-EM visualization of a viral internal ribosome entry site bound to human ribosomes: the IRES functions as an RNA-based translation factor. Cell. 2004 Aug 20;118(4):465-75.

[6] Ayub MJ, Atwood J, Nuccio A, Tarleton R, Levin MJ. Proteomic analysis of the Trypanosoma cruzi ribosomal proteins. Biochem Biophys Res Commun. 2009 Apr 24;382(1):30-4.

[7] Atwood JA, 3rd, Weatherly DB, Minning TA, Bundy B, Cavola C, Opperdoes FR, et al. The Trypanosoma cruzi proteome. Science. 2005 Jul 15;309(5733):473-6.

[8] Beckmann R, Spahn CM, Eswar N, Helmers J, Penczek PA, Sali A, et al. Architecture of the protein-conducting channel associated with the translating 80S ribosome. Cell. 2001 Nov 2;107(3):361-72.

[9] Link AJ, Eng J, Schieltz DM, Carmack E, Mize GJ, Morris DR, et al. Direct analysis of protein complexes using mass spectrometry. Nat Biotechnol. 1999 Jul;17(7):676-82.

[10] Sengupta J, Nilsson J, Gursky R, Spahn CM, Nissen P, Frank J. Identification of the versatile scaffold protein RACK1 on the eukaryotic ribosome by cryo-EM. Nat Struct Mol Biol. 2004 Oct;11(10):957-62.

[11] Liljas A. Comparative biochemistry and biophysics of ribosomal proteins. Int Rev Cytol. 1991;124:103-36.

[12] Uchiumi T, Kominami R. Binding of mammalian ribosomal protein complex P0.P1.P2 and protein L12 to the GTPase-associated domain of $28 \mathrm{~S}$ ribosomal RNA and effect on the accessibility to anti-28 S RNA autoantibody. J Biol Chem. 1997 Feb 7;272(6):3302-8.

[13] Gonzalo P, Lavergne JP, Reboud JP. Pivotal role of the P1 N-terminal domain in the assembly of the mammalian ribosomal stalk and in the proteosynthetic activity. J Biol Chem. 2001 Jun 8;276(23):19762-9.

[14] Planta RJ, Mager WH. The list of cytoplasmic ribosomal proteins of Saccharomyces cerevisiae. Yeast. 1998 Mar 30;14(5):471-7.

[15] Guarinos E, Remacha M, Ballesta JP. Asymmetric interactions between the acidic P1 and P2 proteins in the Saccharomyces cerevisiae ribosomal stalk. J Biol Chem. $2001 \mathrm{Aug}$ 31;276(35):32474-9.

[16] Levin MJ, Vazquez M, Kaplan D, Schijman AG. The Trypanosoma cruzi ribosomal P protein family: classification and antigenicity. Parasitol Today. 1993 Oct;9(10):381-4.

[17] Juri Ayub M, Smulski CR, Nyambega B, Bercovich N, Masiga D, Vazquez MP, et al. Protein-protein interaction map of the Trypanosoma cruzi ribosomal P protein complex. Gene. 2005 Sep 12;357(2):129-36.

[18] Ayub MJ, Barroso JA, Levin MJ, Aguilar CF. Preliminary structural studies of the hydrophobic ribosomal P0 protein from Trypanosoma cruzi, a part of the P0/P1/P2 complex. Protein Pept Lett. 2005 Aug;12(6):521-5. 
[19] Smulski CR, Longhi SA, Ayub MJ, Edreira MM, Simonetti L, Gomez KA, et al. Interaction map of the Trypanosoma cruzi ribosomal P protein complex (stalk) and the elongation factor 2. J Mol Recognit. 2010 Mar-Apr;24(2):359-70.

[20] Tsurugi K, Mitsui K. Bilateral hydrophobic zipper as a hypothetical structure which binds acidic ribosomal protein family together on ribosomes in yeast Saccharomyces cerevisiae. Biochem Biophys Res Commun. 1991 Feb 14;174(3):1318-23.

[21] Santos C, Ballesta JP. The highly conserved protein P0 carboxyl end is essential for ribosome activity only in the absence of proteins P1 and P2. J Biol Chem. 1995 Sep 1;270(35):20608-14.

[22] Lalioti VS, Perez-Fernandez J, Remacha M, Ballesta JP. Characterization of interaction sites in the Saccharomyces cerevisiae ribosomal stalk components. Mol Microbiol. 2002 Nov;46(3):719-29.

[23] Perez-Fernandez J, Remacha M, Ballesta JP. The acidic protein binding site is partially hidden in the free Saccharomyces cerevisiae ribosomal stalk protein P0. Biochemistry. 2005 Apr 12;44(14):5532-40.

[24] Naganuma T, Nomura N, Yao M, Mochizuki M, Uchiumi T, Tanaka I. Structural basis for translation factor recruitment to the eukaryotic/archaeal ribosomes. J Biol Chem. 2010 Feb 12;285(7):4747-56.

[25] Rodriguez-Gabriel MA, Remacha M, Ballesta JP. The RNA interacting domain but not the protein interacting domain is highly conserved in ribosomal protein P0. J Biol Chem. 2000 Jan 21;275(3):2130-6.

[26] Aruna K, Chakraborty T, Rao PN, Santos C, Ballesta JP, Sharma S. Functional complementation of yeast ribosomal P0 protein with Plasmodium falciparum P0. Gene. 2005 Aug 29;357(1):9-17.

[27] Levin MJ, Mesri E, Benarous R, Levitus G, Schijman A, Levy-Yeyati P, et al. Identification of major Trypanosoma cruzi antigenic determinants in chronic Chagas' heart disease. Am J Trop Med Hyg. 1989 Nov;41(5):530-8.

[28] Mesri EA, Levitus G, Hontebeyrie-Joskowicz M, Dighiero G, Van Regenmortel MH, Levin MJ. Major Trypanosoma cruzi antigenic determinant in Chagas' heart disease shares homology with the systemic lupus erythematosus ribosomal P protein epitope. J Clin Microbiol. 1990 Jun;28(6):1219-24.

[29] Kaplan D, Ferrari I, Bergami PL, Mahler E, Levitus G, Chiale P, et al. Antibodies to ribosomal $\mathrm{P}$ proteins of Trypanosoma cruzi in Chagas disease possess functional autoreactivity with heart tissue and differ from anti-P autoantibodies in lupus. Proc Natl Acad Sci U S A. 1997 Sep 16;94(19):10301-6.

[30] Juri Ayub M, Nyambega B, Simonetti L, Duffy T, Longhi SA, Gómez KA, et al. Selective blockade of trypanosomatid protein synthesis by a recombinant antibody antiTrypanosoma cruzi P2ß proteinP2ß protein. PLoS One. 2012;7(5):e36233.

[31] Smulski C, Labovsky V, Levy G, Hontebeyrie M, Hoebeke J, Levin MJ. Structural basis of the cross-reaction between an antibody to the Trypanosoma cruzi ribosomal P2beta protein and the human beta1 adrenergic receptor. FASEB J. 2006 Jul;20(9):1396-406.

[32] Mahler E, Sepulveda P, Jeannequin O, Liegeard P, Gounon P, Wallukat G, et al. A monoclonal antibody against the immunodominant epitope of the ribosomal P2beta 
protein of Trypanosoma cruzi interacts with the human beta 1-adrenergic receptor. Eur J Immunol. 2001 Jul;31(7):2210-6.

[33] Zampieri S, Mahler M, Bluthner M, Qiu Z, Malmegrim K, Ghirardello A, et al. Recombinant anti-P protein autoantibodies isolated from a human autoimmune library: reactivity, specificity and epitope recognition. Cell Mol Life Sci. 2003 Mar;60(3):588-98.

[34] Pizarro JC, Boulot G, Bentley GA, Gomez KA, Hoebeke J, Hontebeyrie M, et al. Crystal Structure of the Complex mAb 17.2 and the C-Terminal Region of Trypanosoma cruzi P2beta Protein: Implications in Cross-Reactivity. PLoS Negl Trop Dis. 2011 Nov;5(11):e1375.

[35] Hastings KE. SL trans-splicing: easy come or easy go? Trends Genet. 2005 Apr;21(4):2407.

[36] Douris V, Telford MJ, Averof M. Evidence for multiple independent origins of transsplicing in Metazoa. Mol Biol Evol. Mar;27(3):684-93.

[37] Derelle R, Momose T, Manuel M, Da Silva C, Wincker P, Houliston E. Convergent origins and rapid evolution of spliced leader trans-splicing in metazoa: insights from the ctenophora and hydrozoa. RNA. Apr;16(4):696-707.

[38] Bangs JD, Crain PF, Hashizume T, McCloskey JA, Boothroyd JC. Mass spectrometry of mRNA cap 4 from trypanosomatids reveals two novel nucleosides. J Biol Chem. 1992 May 15;267(14):9805-15.

[39] Zeiner GM, Sturm NR, Campbell DA. The Leishmania tarentolae spliced leader contains determinants for association with polysomes. J Biol Chem. 2003 Oct 3;278(40):38269-75.

[40] Zamudio JR, Mittra B, Campbell DA, Sturm NR. Hypermethylated cap 4 maximizes Trypanosoma brucei translation. Mol Microbiol. 2009 Jun;72(5):1100-10.

[41] Dhalia R, Reis CR, Freire ER, Rocha PO, Katz R, Muniz JR, et al. Translation initiation in Leishmania major: characterisation of multiple eIF4F subunit homologues. Mol Biochem Parasitol. 2005 Mar;140(1):23-41.

[42] Joshi B, Lee K, Maeder DL, Jagus R. Phylogenetic analysis of eIF4E-family members. BMC Evol Biol. 2005;5:48.

[43] Yoffe Y, Zuberek J, Lerer A, Lewdorowicz M, Stepinski J, Altmann M, et al. Binding specificities and potential roles of isoforms of eukaryotic initiation factor $4 \mathrm{E}$ in Leishmania. Eukaryot Cell. 2006 Dec;5(12):1969-79.

[44] Altmann M, Muller PP, Pelletier J, Sonenberg N, Trachsel H. A mammalian translation initiation factor can substitute for its yeast homologue in vivo. J Biol Chem. 1989 Jul 25;264(21):12145-7.

[45] Robalino J, Joshi B, Fahrenkrug SC, Jagus R. Two zebrafish eIF4E family members are differentially expressed and functionally divergent. J Biol Chem. 2004 Mar 12;279(11):10532-41.

[46] Hernandez G, Altmann M, Sierra JM, Urlaub H, Diez del Corral R, Schwartz P, et al. Functional analysis of seven genes encoding eight translation initiation factor 4E (eIF4E) isoforms in Drosophila. Mech Dev. 2005 Apr;122(4):529-43.

[47] Rodriguez CM, Freire MA, Camilleri C, Robaglia C. The Arabidopsis thaliana cDNAs coding for eIF4E and eIF(iso)4E are not functionally equivalent for yeast 
complementation and are differentially expressed during plant development. Plant J. 1998 Feb;13(4):465-73.

[48] Abascal F, Zardoya R, Posada D. ProtTest: selection of best-fit models of protein evolution. Bioinformatics. 2005 May 1;21(9):2104-5.

[49] Hordijk W, Gascuel O. Improving the efficiency of SPR moves in phylogenetic tree search methods based on maximum likelihood. Bioinformatics. 2005 Dec 15;21(24):433847.

[50] Freire ER, Dhalia R, Moura DM, da Costa Lima TD, Lima RP, Reis CR, et al. The four trypanosomatid eIF4E homologues fall into two separate groups, with distinct features in primary sequence and biological properties. Mol Biochem Parasitol. Mar;176(1):25-36.

[51] Li L, Wang CC. Identification in the ancient protist Giardia lamblia of two eukaryotic translation initiation factor $4 \mathrm{E}$ homologues with distinctive functions. Eukaryot Cell. 2005 May;4(5):948-59.

[52] Barhoumi M, Tanner NK, Banroques J, Linder P, Guizani I. Leishmania infantum LeIF protein is an ATP-dependent RNA helicase and an eIF4A-like factor that inhibits translation in yeast. FEBS J. 2006 Nov;273(22):5086-100.

[53] Dhalia R, Marinsek N, Reis CR, Katz R, Muniz JR, Standart N, et al. The two eIF4A helicases in Trypanosoma brucei are functionally distinct. Nucleic Acids Res. 2006;34(9):2495-507.

[54] Blum S, Mueller M, Schmid SR, Linder P, Trachsel H. Translation in Saccharomyces cerevisiae: initiation factor 4A-dependent cell-free system. Proc Natl Acad Sci U S A. 1989 Aug;86(16):6043-6.

[55] Li W, Belsham GJ, Proud CG. Eukaryotic initiation factors 4A (eIF4A) and 4G (eIF4G) mutually interact in a 1:1 ratio in vivo. J Biol Chem. 2001 Aug 3;276(31):29111-5.

[56] Kressler D, de la Cruz J, Rojo M, Linder P. Fal1p is an essential DEAD-box protein involved in 40S-ribosomal-subunit biogenesis in Saccharomyces cerevisiae. Mol Cell Biol. 1997 Dec;17(12):7283-94.

[57] Shibuya T, Tange TO, Sonenberg N, Moore MJ. eIF4AIII binds spliced mRNA in the exon junction complex and is essential for nonsense-mediated decay. Nat Struct Mol Biol. 2004 Apr;11(4):346-51.

[58] Yoffe Y, Leger M, Zinoviev A, Zuberek J, Darzynkiewicz E, Wagner G, et al. Evolutionary changes in the Leishmania eIF4F complex involve variations in the eIF4EeIF4G interactions. Nucleic Acids Res. 2009 Jun;37(10):3243-53.

[59] Hernandez G. On the origin of the cap-dependent initiation of translation in eukaryotes. Trends Biochem Sci. 2009 Apr;34(4):166-75.

[60] Uchiumi T, Hori K, Nomura T, Hachimori A. Replacement of L7/L12.L10 protein complex in Escherichia coli ribosomes with the eukaryotic counterpart changes the specificity of elongation factor binding. J Biol Chem. 1999 Sep 24;274(39):27578-82.

[61] Perentesis JP, Phan LD, Gleason WB, LaPorte DC, Livingston DM, Bodley JW. Saccharomyces cerevisiae elongation factor 2. Genetic cloning, characterization of expression, and G-domain modeling. J Biol Chem. 1992 Jan 15;267(2):1190-7.

[62] Ryazanov AG. Ca2+/calmodulin-dependent phosphorylation of elongation factor 2. FEBS Lett. 1987 Apr 20;214(2):331-4. 
[63] Ryazanov AG, Shestakova EA, Natapov PG. Phosphorylation of elongation factor 2 by EF-2 kinase affects rate of translation. Nature. 1988 Jul 14;334(6178):170-3.

[64] Montanaro L, Sperti S, Testoni G, Mattioli A. Effect of elongation factor 2 and of adenosine diphosphate-ribosylated elongation factor 2 on translocation. Biochem J. 1976 Apr 15;156(1):15-23.

[65] MacConnell WP, Kaplan NO. The activity of the acidic phosphoproteins from the $80 \mathrm{~S}$ rat liver ribosome. J Biol Chem. 1982 May 25;257(10):5359-66.

[66] Gomez-Lorenzo MG, Spahn CM, Agrawal RK, Grassucci RA, Penczek P, Chakraburtty $\mathrm{K}$, et al. Three-dimensional cryo-electron microscopy localization of EF2 in the Saccharomyces cerevisiae $80 S$ ribosome at 17.5 A resolution. EMBO J. 2000 Jun 1;19(11):2710-8.

[67] Bargis-Surgey P, Lavergne JP, Gonzalo P, Vard C, Filhol-Cochet O, Reboud JP. Interaction of elongation factor eEF-2 with ribosomal P proteins. Eur J Biochem. 1999 Jun;262(2):606-11.

[68] Shastry M, Nielsen J, Ku T, Hsu MJ, Liberator P, Anderson J, et al. Species-specific inhibition of fungal protein synthesis by sordarin: identification of a sordarin-specificity region in eukaryotic elongation factor 2. Microbiology. $2001 \mathrm{Feb} ; 147(\mathrm{Pt} 2): 383-90$.

[69] Santos C, Rodriguez-Gabriel MA, Remacha M, Ballesta JP. Ribosomal P0 protein domain involved in selectivity of antifungal sordarin derivatives. Antimicrob Agents Chemother. 2004 Aug;48(8):2930-6.

[70] Scory S, Steverding D. Differential toxicity of ricin and diphtheria toxin for bloodstream forms of Trypanosoma brucei. Mol Biochem Parasitol. 1997 Dec 1;90(1):289-95.

[71] Juri Ayub M, Ma KW, Shaw PC, Wong KB. Trypanosoma cruzi: high ribosomal resistance to trichosanthin inactivation. Exp Parasitol. 2008 Mar;118(3):442-7.

[72] Sprang SR. G protein mechanisms: insights from structural analysis. Annu Rev Biochem. 1997;66:639-78.

[73] Kelley LA, Sternberg MJ. Protein structure prediction on the Web: a case study using the Phyre server. Nat Protoc. 2009;4(3):363-71.

[74] Jorgensen R, Ortiz PA, Carr-Schmid A, Nissen P, Kinzy TG, Andersen GR. Two crystal structures demonstrate large conformational changes in the eukaryotic ribosomal translocase. Nat Struct Biol. 2003 May;10(5):379-85.

[75] Mishra J, Saxena A, Singh S. Chemotherapy of leishmaniasis: past, present and future. Curr Med Chem. 2007;14(10):1153-69. 